\title{
IV. On some problems connected with the flow of electricity in a plane
}

\section{Oliver J. Lodge B.Sc.}

To cite this article: Oliver J. Lodge B.Sc. (1876) IV. On some problems connected with the flow of electricity in a plane, Philosophical Magazine Series 5, 2:8, 37-47, DOI:

$10.1080 / 14786447608639157$

To link to this article: http://dx.doi.org/10.1080/14786447608639157

曲 Published online: 13 May 2009.

Submit your article to this journal \lceil

Џ Article views: 1

Q View related articles $₫$ 


\section{[ 37 ]}

IV. On some Problems connected with the Filow of Electricity in a Plane. By Ourver J. Lodge, B.Sc.

[Continued from vol. i. p. 389.]

General form of the resistance-expression for two poles.

$\S 22 . T T$ is convenient at this point to notice the general 1 form of the resistance expression for two poles $A$ and $B$ in any bounded plate. It may always be written in the form

$$
\frac{c}{\pi \kappa \delta} \log \left(\frac{\mathrm{AB}}{\rho} \cdot \mathrm{Q}\right), \text {. . . . . }
$$

where $Q$ is a numerical quantity which depends on the general form of the boundaries of the plate, and on the position of the poles with respect to those boundaries; while $c$ is a number which depends only on that part of the boundary which is infinitely near a pole. As long as the poles are not placed on the edge of the plate, $c=1$ and is unaffected by any change in the boundary; but if either pole is placed on the edge, the angle on which it is placed determines $c$,-the general law being that if $\mathrm{A}$ is on an angle $\frac{\pi}{m}$, and $\mathrm{B}$ on an angle $\frac{\pi}{n}$, then $c=m+n$. (When both poles are inside the plate, both angles equal $\mathbf{2 \pi}$, and therefore $c=1$, as stated above.)

It is unnecessary to prove this rule formally ; but it is a consequence of the necessary condition that $A$ and $B$ must be of equal strength in the limited sheet; for this condition requires that if they are on angles $\frac{2 \pi}{2 m}$ and $\frac{2 \pi}{2 n}$. respectively, their strengths in the unlimited sheet must be in the ratio of $2 m: 2 n$, and accordingly the quantity $\frac{A B}{\rho}$ in $(\alpha) \S 8$ will occur to the $2(m+n)$ th power.

Hence, in the process of finding the resistance of any plane conductor containing two small electrodes, the determination of $\mathrm{Q}$ is the only difficulty. We have found it for the general polygon of two sides; let us proceed to find it for a fow triangles.

Resistance of an isosceles right-angled triangle. Poles on the equal angles.

$\S 23$. This case was in reality the first figure with rectilinear boundaries which I attempted. The positions of the images of $A$ are shown in fig. 5 ; they are on the corners of squares covering all the plane. The images of $\mathrm{B}$ will be similarly arranged, every source being surrounded by four sinks. 
To determine the value of the product $\left(\frac{\mathrm{AB}}{\rho} \cdot \mathrm{Q}\right)$ in the resistance-expression $(\beta)(\S 8)$, take the images in rows, and find the product for each row separately (cf. $\S 9$ ). Call the line $A B$,

Fig. 5.

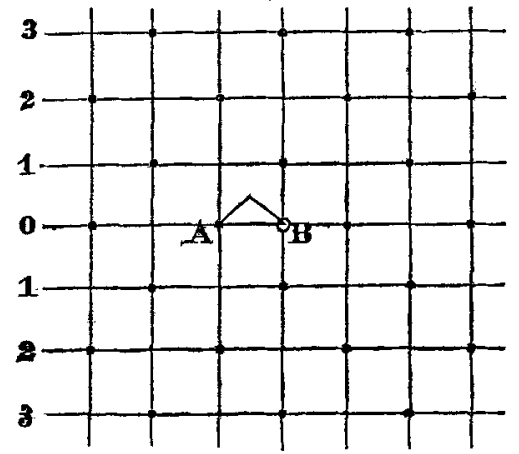

Images of the pole $A$ in an isosceles right-angled triangle.

fig. 5 , row 0 ; the two rows one on either side of $A B$ call rows 1 ; row 2 will be the row next beyond each of these; and so on, the images being evidently symmetrical with respect to the line $A B$. The whole product $Q$ will then equal $Q_{0}, Q_{1}^{2}, Q_{2}^{2} \ldots$, where $Q_{x}$ stands for the product of the $\frac{A_{x} B}{A_{x} A}$ 's in one row $x$. From the figure we see that

$$
\begin{aligned}
& \mathrm{Q}_{0}=\frac{1.3 .3 .5 .5 .7 \ldots}{2.2 .4 .4 .6 .6 \ldots} \\
& =\frac{2}{\pi}=\frac{1}{\sigma(0)}, \\
& \mathrm{Q}_{1}^{2}=\frac{1^{2}}{1^{2}+1^{2}} \cdot \frac{2^{2}+1^{2}}{1^{2}+1^{2}} \cdot \frac{2^{2}+1^{2}}{3^{2}+1^{2}} \cdot \frac{4^{2}+1^{2}}{3^{2}+1^{2}} \cdot \frac{4^{2}+1^{2}}{5^{2}+1^{2}} \ldots=1^{2} \cdot \sigma^{2}(1), \\
& Q_{2}^{2}=\frac{1^{2}+2^{2}}{2^{2}} \cdot \frac{1^{2}+2^{2}}{2^{2}+2^{2}} \cdot \frac{3^{2}+2^{2}}{2^{2}+2^{2}} \cdot \frac{3^{2}+2^{2}}{4^{2}+2^{2}} \ldots \quad=\frac{1}{2^{2} \varpi^{2}(2)}, \\
& Q_{3}^{2}=\frac{3^{2}}{1^{2}+3^{2}} \cdot \frac{2^{2}+3^{2}}{1^{2}+3^{2}} \cdot \frac{2^{2}+3^{2}}{3^{2}+3^{2}} \ldots \\
& =3^{2} \varpi^{2}(3), \\
& \mathrm{Q}_{x}^{ \pm 1}=x \varpi(x),
\end{aligned}
$$

where the index is to be taken positive when $x$ is odd, negative when $x$ is even ; and where $\varpi(x)$ stands for the product

$$
\frac{2^{2}+x^{2}}{1^{2}+x^{2}} \cdot \frac{4^{2}+x^{2}}{3^{2}+x^{2}} \cdot \frac{6^{2}+x^{2}}{5^{2}+x^{2}} \cdots \frac{1}{\sqrt{ }\left(\infty^{2}+x^{2}\right)},
$$


of which Wallis's expression is the particular case when $x=0$.

The entire product $Q$ also shows an evident resemblance to Wallis's form, being

$$
\mathrm{Q}=\frac{2}{\pi}\left(\frac{1 \varpi(1) \cdot 3 \varpi(3) \ldots}{2 \sigma(2) \cdot 4 \varpi(4) \ldots}\right)^{2}
$$

Further than this I was unable to proceed; so I showed the products to Mr. J. W. L. Glaisher during the Bristol Meeting of the British Association; and he very kindly told me how to evaluate the product denoted above by $\sigma(x)$, and subsequently worked out the compound product $Q$. Taking the well-known trigonometrical identities

$$
\begin{aligned}
& \sin \frac{\pi x}{2}=\frac{\pi x}{2}\left(1-\frac{x^{2}}{2^{2}}\right)\left(1-\frac{x^{2}}{4^{2}}\right)\left(1-\frac{x^{2}}{6^{2}}\right) \ldots, \\
& \cos \frac{\pi x}{2}=\left(1-\frac{x^{2}}{1^{2}}\right)\left(1-\frac{x^{2}}{3^{2}}\right)\left(1-\frac{x^{2}}{5^{2}}\right) \ldots,
\end{aligned}
$$

Mr. Glaisher divided one by the other after putting $x \sqrt{-1}$ for $x$; he thus obtained

$$
\begin{aligned}
\tan \frac{i \pi x}{2} & =\frac{i \pi x}{2} \cdot \frac{2^{2}+x^{2}}{1^{2}+x^{2}} \cdot \frac{4^{2}+x^{2}}{3^{2}+x^{2}} \ldots \frac{1^{2} \cdot 3^{2} \cdot 5^{2} \ldots}{2^{2} \cdot 4^{2} \cdot 6^{2} \ldots} \\
& =\frac{i \pi x}{2} \cdot \varpi(x) \cdot \frac{2}{\pi}
\end{aligned}
$$

whence

$$
x \varpi(x)=\frac{1}{i} \tan \frac{i \pi x}{2}=\tanh \frac{\pi x}{2} *
$$

This result gives us

$$
Q=\frac{2}{\pi}\left(\frac{\tanh \frac{\pi}{2} \cdot \tanh \frac{3 \pi}{2} \cdots}{\tanh \frac{2 \pi}{2} \cdot \tanh \frac{4 \pi}{2} \ldots}\right)^{2}
$$

The part inside the brackets may be written

$$
\sqrt{\frac{\pi Q}{2}}=\frac{1-e^{-\pi}}{1+e^{-\pi}} \cdot \frac{1-e^{-3 \pi}}{1+e^{-3 \pi}} \cdots \frac{1+e^{-2 \pi}}{1-e^{-2 \pi}} \cdot \frac{1+e^{-4 \pi}}{1-e^{-4 \pi}} \cdots ;
$$

and this Mr. Glaisher saw at once was a special case of Jacobi's products; two of which (being those required in this paper) I write down here-

* Mr. Glaisher also obtained an expression for the more general product $\frac{2^{n}+x^{n}}{1^{n}+x^{n}} \cdot \frac{4^{n}+x^{n}}{3^{n}+x^{n}}, \ldots$, which he has communicated to the Mathematieal Society. 


$$
\begin{aligned}
& \frac{1-q}{1+q} \cdot \frac{1-q^{3}}{1+q^{3}} \cdot \frac{1-q^{5}}{1+q^{5}} \ldots=k^{\frac{1}{4}}, \quad . \quad . \quad . \\
& \frac{1-q^{2}}{1+q^{2}} \cdot \frac{1-q^{4}}{1+q^{4}} \cdot \frac{1-q^{6}}{1+q^{6}} \ldots=\left(\frac{2 \sqrt{k^{\prime}} \mathrm{K}}{\pi}\right)^{\frac{1}{2}} ; .
\end{aligned}
$$

$q$ stands for $e^{-\pi \frac{\mathbf{K}^{\prime}}{\mathbf{K}}}, \mathrm{K}$ having its ordinary meaning of

$$
\int_{0}^{\frac{\pi}{2}} \frac{d \theta}{\sqrt{ }\left(1-k^{2} \sin ^{2} \theta\right)}
$$

Taking the modulus-angle $45^{\circ}$ so that $k=k^{\prime}=\frac{1}{\sqrt{2}}$ and $\mathrm{K}=\mathrm{K}^{\prime}=\mathrm{K}_{0}$ say, and then dividing $(\delta)$ by $(\epsilon)$, there results the product required,

$$
\sqrt{\frac{\pi \mathrm{Q}}{2}}=\sqrt{\frac{\pi}{2 \mathrm{~K}_{0}}}
$$

Hence $Q=\frac{1}{\mathrm{~K}_{0}}$, and the resistance of a triangle with each electrode on an angle of $45^{\circ}$ is

$$
\mathrm{R}=\frac{8}{\pi \kappa \delta} \log \left(\frac{\mathrm{AB}}{\rho} \cdot \frac{1}{\mathrm{~K}_{0}}\right) . . . .
$$

The numerical value of $K_{0}$ from Legendre's Tables is

$$
1 \cdot 854074677301 \text {. }
$$

$\S 24$. If one writes out (17) in two parts,

$$
\frac{8}{\pi \kappa \delta} \log \frac{\mathrm{AB}}{\rho}-\frac{8}{\pi \kappa \delta} \log \int_{0}^{\frac{\pi}{2}} \frac{d \theta}{\sqrt{ }\left(1-\frac{1}{2} \sin ^{2} \theta\right)}, .
$$

the first term is the resistance of a segment of a circle with base $A B$ and with its are touching the two sides of the triangle at $A$ and $B$; and the second term may be called the effect of the "coquadrant" * by which the triangle exceeds the segment in area. But one must not imagine that this term expresses the resistance of this figure. The fact is that its resistance cannot be found by any consideration of $(17)^{\prime}$, for reasons which shall be stated, $\S 30$, footnote. It is worth while to notice the arrangement of poles and flow-lines which would produce this coquadrant figure in an unlimited sheet; but they will come better later.

* I call it a coquadrant because it is the excess of a square over the quadrant whose radius is a side of the square: more generally the figure enclosed by any circular arc and two tangents might be called a cosector; it would be the excess of an isosceles cirquadrilateral over the inscribed sector, and it only differs from a sector in having the equal angles $0^{\circ}$ instead of $90^{\circ}$. 
with the Flow of Electricity in a Plane.

Resistance of an equilateral triangle. Poles on two of the angles.

$\S 25$. The images of $\mathrm{A}$ for this case are shown in fig. 6 ,

Fig. 6.

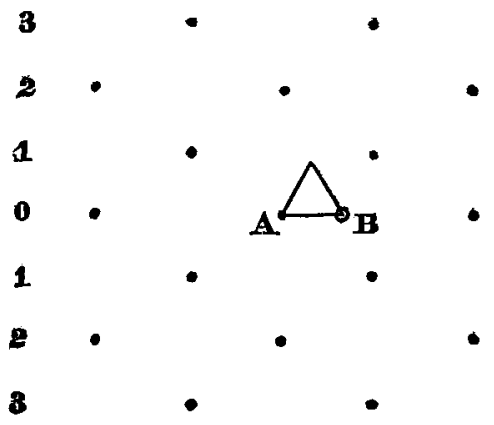

Images of the pole $A$ in an equilateral triangle.

being on the vertices of equilateral triangles $\sqrt{3}$ times the linear dimensions of the original one. Take the images in rows as before $(\$ 23)$, calling the line A B row 0 , and find the product $Q_{x}$ for each row separately.

$$
\begin{aligned}
& \mathrm{Q}_{0}=\frac{2.4 \cdot 5 \cdot 7.8 \cdot 10 \ldots}{3.3 .6 \cdot 6.9 \cdot 9 \ldots} \quad=\frac{3 \sqrt{\mathfrak{3}}}{2 \pi}=\sqrt{0^{2} \phi(0) \psi(0)}, \\
& \mathrm{Q}_{1}^{2}=\frac{1^{2}+3}{3^{2}+3} \cdot \frac{5^{2}+3}{3^{2}+3} \cdot \frac{7^{2}+3}{9^{2}+3} \cdot \frac{11^{2}+3}{9^{2}+3} \cdot \frac{13^{2}+3}{15^{2}+3} \ldots=\phi(\sqrt{3}), \\
& \mathrm{Q}_{2}^{2}=\frac{2^{2}+2^{2} .3}{2^{2} \cdot 3} \cdot \frac{4^{2}+2^{2} \cdot 3}{6^{2}+2^{2} \cdot 3} \cdot \frac{8^{2}+2^{2} .3}{6^{2}+2^{2} .3} \cdot \frac{10^{2}+2^{2} .3}{12^{2}+2^{2} .3} \ldots=\psi(2 \sqrt{3}) \text {, } \\
& \mathrm{Q}_{3}^{2}=\frac{1^{2}+3^{2} .3}{3^{2}+3^{2} .3} \cdot \frac{5^{2}+3^{2} \cdot 3}{3^{2}+3^{2} .3} \cdot \frac{7^{2}+3^{2} .3}{9^{2}+3^{2} .3} \ldots, \quad=\phi(3 \sqrt{3}), \\
& \mathrm{Q}=\frac{3 \sqrt{3}}{2 \pi} \phi(\sqrt{3}) \psi(2 \sqrt{3}) \phi(3 \sqrt{3}) \psi(4 \sqrt{3}) \ldots
\end{aligned}
$$

Now

$$
\begin{aligned}
\phi(x)=\frac{1^{2}+x^{2}}{3^{2}+x^{2}} \cdot \frac{5^{2}+x^{2}}{3^{2}+x^{2}} \cdot \frac{7^{2}+x^{2}}{9^{2}+x^{2}} \ldots= & \frac{1}{4} \cdot \frac{\cosh \frac{\pi x}{2}}{\cosh ^{3} \frac{\pi x}{6}} \\
= & \frac{1+3 \tanh ^{2} \frac{\pi x}{6}}{4}
\end{aligned}
$$


this result being evident if numerator and denominator are multiplied by

and similarly

$$
3^{2}+x^{2} .9^{2}+x^{2} .15^{2}+x^{2} \ldots ;
$$

$$
\begin{aligned}
\psi(x)=\frac{2^{2}+x^{2}}{x^{2}} \cdot \frac{4^{2}+x^{2}}{6^{2}+x^{2}} \cdot \frac{8^{2}+x^{2}}{6^{2}+x^{2}} \ldots= & \frac{1}{4} \cdot \frac{\sinh \frac{\pi x}{2}}{\sinh ^{3} \frac{\pi x}{6}} \\
& =\frac{1+3 \operatorname{coth}^{2} \frac{\pi x}{6}}{4} .
\end{aligned}
$$

Hence

$$
\begin{array}{r}
\mathrm{Q}=\frac{3 \sqrt{3}}{2 \pi} \cdot \frac{1+3 \tanh ^{2} \frac{\pi}{2 \sqrt{3}}}{4} \cdot \frac{1+3 \tanh ^{2} \frac{3 \pi}{2 \sqrt{3}}}{4} \cdot \frac{1+3 \tanh ^{2} \frac{5 \pi}{2 \sqrt{3}}}{4} \ldots \\
\quad \times \frac{1+3 \operatorname{coth}^{2} \frac{2 \pi}{2 \sqrt{3}}}{4} \cdot \frac{1+3 \operatorname{coth}^{2} \frac{4 \pi}{2 \sqrt{3}}}{4} \cdot \frac{1+3 \operatorname{coth}^{2} \frac{6 \pi}{2 \sqrt{3}}}{4} \ldots
\end{array}
$$

This product I sent to Mr. Glaisher; and he returned it reduced to a product of two theta-functions. He proved the two following identities :-

$$
\begin{aligned}
\mathrm{P}_{1}=\frac{1+m \tanh ^{2} \theta}{1+m} \cdot \frac{1+m \tanh ^{2} 3 \theta}{1+m} \cdot \frac{1+m \tanh ^{2} 5 \theta}{1+m} \ldots \\
=\sqrt{\frac{\pi}{2 \mathrm{~K}} \Theta\left(\frac{2 \mathrm{~K} x}{\pi}\right)}
\end{aligned}
$$

and

where

$$
\begin{aligned}
\mathrm{P}_{2}=\frac{1+m \operatorname{coth}^{2} 2 \theta}{1+m} & \cdot \frac{1+m \operatorname{coth}^{2} 4 \theta}{1+m} \cdot \frac{1+m \operatorname{coth}^{2} 6 \theta}{1+m} \ldots \\
& =\frac{1}{\cos x} \sqrt{\frac{\pi^{3}}{(2 \mathrm{~K})^{3} k k^{\prime}}} \cdot \mathrm{H}\left(\frac{2 \mathrm{~K} x}{\pi}+\mathrm{K}\right),
\end{aligned}
$$

$$
\cos 2 x=\frac{m-1}{m+1} \text { and } 2 \theta=\pi \frac{\mathbf{K}^{\prime}}{\bar{K}} \text {. }
$$

In our present case

so

$$
m=3 \text { and } \theta=\frac{\pi}{2 \sqrt{3}} ;
$$

$$
\mathrm{K}=\sqrt{3} \mathrm{~K}^{\prime}, k=\sin 75^{\circ} \text {, and } x=\frac{\pi}{6} .
$$


Hence

and

$$
\mathrm{P}_{1}=\sqrt{\frac{\pi}{2 \mathrm{~K}}} \Theta\left(\frac{1}{3} \mathrm{~K}\right), \quad \frac{\sqrt{3}}{2} \mathrm{P}_{2}=\sqrt{\frac{\pi^{3}}{8 \mathrm{~K}^{3} k k^{\prime}}} \mathrm{H}\left(\frac{2}{3} \mathrm{~K}\right),
$$

$$
\mathrm{Q}=\frac{3 \sqrt{3}}{2 \pi} \mathrm{P}_{1} \mathrm{P}_{2}=\frac{3 \pi}{4 \mathrm{~K}^{2} \sqrt{k^{\prime}}} \Theta\left(30^{\circ}\right) \Theta_{1}\left(60^{\circ}\right)\left(\bmod . \text { angle } 75^{\circ}\right),
$$

writing $\Theta_{1}(x)$ for $\frac{1}{\sqrt{k}} \mathrm{H}(x)$.

From the British-Association Tables

$$
\begin{aligned}
& \log \frac{1}{K^{2} \sqrt{k^{\prime}}}=\overline{1} \cdot 4091498986, \\
& \log \Theta\left(30^{\circ}\right)=\overline{1} \cdot 9223413942, \\
& \log \Theta_{1}\left(60^{\circ}\right)=\cdot 0491498189 ;
\end{aligned}
$$

so

or

$$
\log _{10} Q=\overline{1} \cdot 7528522478 \text {, }
$$

$$
\mathrm{Q}=\frac{1}{1 \cdot 76664} \text { about, }
$$

and the resistance of the triangle is

$$
\mathrm{R}=\frac{6}{\pi \kappa \delta} \log \left(\frac{\mathrm{AB}}{\rho} \cdot \mathrm{Q}\right) . . . . .
$$

Manner in which the image-method fails for an angle of $120^{\circ}$.

$\S 26$. We found in $\S 5$ that the only isosceles triangles which could be treated by the method of images were those with the equal angles either $0^{\circ}, 45^{\circ}, 60^{\circ}, 90^{\circ}$, or $180^{\circ}$; and of these all except the first have been already done in the course of this paper $(\$ \S 23,25,19,10)$, and the first shall be considered in the next section. But suppose we attempted the triangle with equal angles $30^{\circ}$, which is apparently a very simple case; the images of the source $A$ (on one of the equal angles) would be readily placed on the corners of regular hexagons; but they would be symmetrically situated with respect to the middle line of the triangle, and hence when the images of the sink B came to be placed they would coincide with those of $\mathrm{A}$ and would blot them all out of the sheet. Or if we overlook this and apply the expression $(\beta)$ to the images of $A$, then, because of their symmetry with respect to the triangle, the product $Q$ ( $\S 8$ \& 22) will equal 1 ; which can be proved to be wrong ( $\$ 27)$. This failure, caused by the presence of the angle $120^{\circ}$, is one that cannot, even apparently, be got over by silvering both 
sides of the boundaries $(\S 4)$; for, in order to arrange poles in an unlimited sheet so as to be symmetrical with respect to all three sides of the triangle, it is necessary that one and the same point shall be at the same time both a source and a sink. Dr. Henrici suggests that this is possible if the plane consists of two leaves, and recommends in general, whenever both sides of a boundary have to be silvered and some of the images are real (that is, occur in the given plate itself), that these real images be put in another leaf of the plane. It seems just possible that some such contrivance might enable the image-method to be applied to polygons whose angles are not integral submultiples of $\pi$.

Empirical formula for the resistance of a general isosceles triangle. Poles on the equal angles.

$\$ 27$. Consider a regular polygon of $n$ sides, with one electrode (radius $\rho$ ), A, fig. 7 , at its centre, and with its entire periphery maintained at one potential. The resistance $\mathrm{R}_{\boldsymbol{n}}$ of such a polygon to radial flow is evidently something between that of its inscribed and that of its circumscribing circle; in other words,

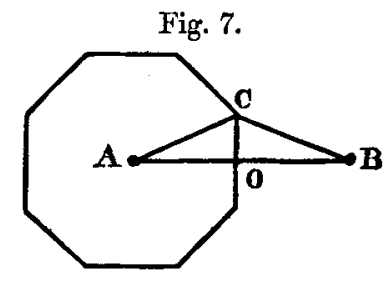

$$
\frac{1}{2 \pi \kappa \delta} \log \frac{\mathrm{AO}}{\rho}<\mathrm{R}_{n}<\frac{1}{2 \pi \kappa \delta} \log \frac{\mathrm{AC}}{\rho} .
$$

Now the resistance $R$ of the isosceles triangle A B C (fig. 7) equals twice the resistance of the triangle $\mathrm{AOC}$, which again equals $2 n$ times the resistanee of the polygon. Hence, writing $\mathrm{AC}=\mathrm{AO} \sec \mathrm{CAO}=\frac{1}{2} \mathrm{AB} \sec \frac{\pi}{n}$,

$$
\frac{2 n}{\pi \kappa \delta} \log \frac{\mathrm{AB}}{2 \rho}<\mathrm{R}<\frac{2 n}{\pi \kappa \delta} \log \left(\frac{\mathrm{AB}}{2 \rho} \sec \frac{\pi}{n}\right) ;
$$

or, calling the angle $\mathrm{CAO} \theta$, we may write the resistance of any isosceles triangle with equal angles $\theta$,

$$
\mathrm{R}=\frac{2}{\theta_{\kappa} \delta} \log \left(\frac{\mathrm{AB}}{\rho} \frac{1}{f(\theta)}\right), \quad . \quad . \quad .
$$

where $f(\theta)$ is something between $2 \cos \theta$ and 2 . Hence the limit of $Q$ when the angles of the triangle vanish is $\frac{1}{2}$. Moreover when the angles are $30^{\circ}, f(\theta)$ must lie between 2 and $\sqrt{3}$; it cannot, therefore, be 1 as the product of images $(\$ 26)$ would lead us to believe.

Looking at the few values of $f(\theta)$ which are known, one 
may notice how nearly they divide the interval allowed to them, $2-2 \cos \theta$, in the ratio $1: 3$. The quantity which actually does this is $\frac{3+\cos \theta}{2}$; and this quantity agrees with the known values of $f(\theta)$ to an extent shown in the Table.

\begin{tabular}{|c|l|l|}
\hline$\theta$. & $f(\theta)$. & $\frac{3+\cos \theta}{2}$ \\
\hline 0 & $2 \cdot 0$ & $2 \cdot 0$ \\
45 & $1 \cdot 854$ & 1.853 \\
60 & $1 \cdot 766$ & 1.75 \\
90 & 1.57 & 1.50 \\
180 & 1.0 & $1 \cdot 0$ \\
\hline
\end{tabular}

The maximum divergence appears to occur about when $\theta=90^{\circ}$.

The empirical formula, then,

$$
\frac{2}{\theta_{\kappa} \delta} \log \left(\frac{\mathrm{AB}}{\rho} \cdot \frac{2}{3+\cos \theta}\right) \text {. . . . . }
$$

gives the resistance of any isosceles triangle with poles on the equal angles $\theta$ with sufficient accuracy for a practical purpose ; and it becomes very accurate indeed when the equal angles are small. The value of $Q$ indicated by it for the case $\theta=30^{\circ}$ is $\frac{1}{1.9330}$; and I venture to think that the real value will be found to differ from this only in the last place of decimals.

The resistance to the flow of electricity from a central electrode of radius $\rho$ to the periphery of a regular polygon of $n$ sides is, to the same degree of approximation as the above,

$$
\frac{1}{2 \pi \kappa \delta} \log \left(\frac{r}{\rho} \cdot \frac{4}{3+\cos \frac{\pi}{n}}\right), \text {. . . . }
$$

$r$ being the radius of the inscribed circle.

$\S 28$. And here I would remark that the thickness $(\delta)$ of the plate in which the flow takes place has never been limited in any way; so the plate may extend itself into a cylinder of any length, provided the point poles extend themselves into lines at the same time. Also, though we are using the language of dynamic electricity only, yet the resistance obtained applies equally well to heat if we substitute hot and cold bodies for sources and sinks, temperature for potential, and isothermals for equipotential lines. Further, by reason of the analogy between conductivity and inductive capacity, we obtain at the 
same time the inductive capacity of a dielectric medium exposed to conductors at different potentials which correspond to the poles in the dynamic problem.

Resistance of some squares and right-angled triangles.

$\$ 29$. The forms assumed by the resistance-expressions $(\$ 8)$ for the case of a square plate with the poles in certain definite positions may be recorded, together with the products which lead to them. The resistance seems always to be of the form

$$
\mathrm{R}=\frac{m+n}{\pi \kappa \delta} \log \left(\frac{\mathrm{AB}}{\rho} \cdot \frac{2^{\alpha}}{\mathrm{K}_{0}}\right), \ldots .
$$

where the poles $A$ and $B$ are on angles $\frac{\pi}{m}$ and $\frac{\pi}{n}$ respectively, and where $\alpha$ takes different values in different cases, and is the only thing which varies. $\mathrm{K}_{0}$ still stands for the first complete elliptic integral with modulus $\frac{1}{\sqrt{2}}$.

For the case represented in fig. 8 the value of $\alpha$ is zero, because the resistance of such a square is just half that of the right-angled triangle (17) §23.

When the poles are as in fig. 9, $\alpha=\frac{1}{4}$; the product in $(\beta)$ which leads to the result consists of terms $\operatorname{coth}^{2} \frac{\pi x}{2}$, where $x$ takes the values $2,4,6, \ldots$.

The case of fig. 10 gives us $\alpha=\frac{1}{2}$, the product being $\Pi\left(\operatorname{coth}^{2} \frac{\pi x}{2}\right)$, with $x$ suceessively $1,2,3,4, \& c$.

The case of fig. 11, being unsymmetrical in sources and sinks, requires the complete expression $(\alpha) \S 8$; and, because the pole at the corner of the square has to be four times as strong as the other in the infinite sheet, the two factors inside the brackets of $(\alpha)$ occur to different powers ; in other words, calling the Fig. 8.

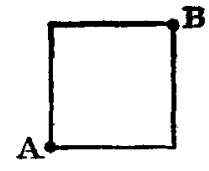

Fig. 9.

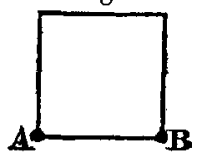

Fig. 10.

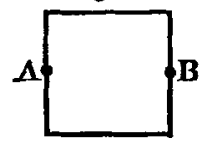

Fig. 11.

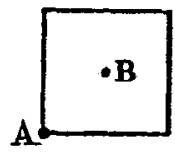
factors $P_{1}$ and $P_{2}$, we shall have

$$
\mathrm{Q}^{5}=\mathrm{P}_{1} \mathrm{P}_{2}^{4}
$$

Now $P_{1}$, the product referring to the images of the pole at the centre, is the old one whose value is $\frac{1}{K_{0}}(\S 23)$. But the pro- 
duct for the images of the corner pole will be found to be

$$
\mathrm{P}_{2}=\frac{2 \sqrt{2}}{\pi} \Pi\left(\operatorname{coth} \frac{\pi x}{2} \operatorname{coth} \frac{\pi x}{4}\right) \Pi\left(\frac{\operatorname{coth} \frac{\pi x^{\prime}}{2}}{\operatorname{coth} \frac{\pi x}{4}}\right),
$$

where $x$ is to take the successive values $4,8,12, \ldots$, and $x^{f}$ the values $2,6,10, \ldots$.

Writing this product $\mathrm{P}_{2}$ in the form

$$
\frac{2 \sqrt{2}}{\pi} \cdot \frac{\tanh \frac{\pi}{2} \cdot \tanh \frac{3 \pi}{2} \ldots}{\tanh \frac{2 \pi}{2} \cdot \tanh \frac{4 \pi}{2} \ldots} \cdot \operatorname{coth} \pi \cdot \operatorname{coth} 2 \pi \cdot \operatorname{coth} 3 \pi \ldots,
$$

it is broaght into connexion with $(\delta)$ and $(\epsilon)(\S 23)$; and its value is thus found to be

Hence

$$
\mathrm{P}_{2}=\frac{2 \vee \overline{2}}{\pi} \sqrt{\frac{\pi}{2 \mathrm{~K}_{0}}} \sqrt{\frac{\pi}{2^{\frac{3}{4}} \mathrm{~K}_{0}}}=\frac{2^{\frac{5}{8}}}{\mathrm{~K}_{0}} .
$$

$$
\mathrm{Q}^{5}=\frac{2^{\frac{3}{2}}}{\mathrm{~K}_{0}^{5}}, \text { or } \mathrm{Q}=\frac{\sqrt{2}}{\mathrm{~K}_{0}}
$$

In other words, the resistance of a square plate with the poles arranged as in fig. 11 is given by (21), if for $m+n$ we read its value $\frac{5}{2}$, and consider $\alpha$ equal to $\frac{1}{2}$.

One more distinct case may be mentioned, namely the right-angled triangle shown in fig. 12 . This also requires two factors raised to different powers; and the result is the expression (21) with $\alpha=\frac{1}{6}$.

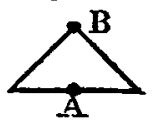

[To be continued.]

V. Some Remarks on the Finite Integration of Linear Partial Differential Equations with constant Coefficients. By the Rev. S. Earnshaw, M.A.

To the Editors of the Philosophical Magazine and Journal. Gentlemer,

TN your Magazine for June 1849 you kindly printed a short paper of mine "On the Transformation of Linear Partial Differential Equations with constant Coefficients to Fundamental Forms," in which I promised to make "in a future communication a few remarks on the finite integration" of equations of the second order with two or three independent 